Check for updates

Cite this: RSC Adv., 2018, 8, 38146

Received 1st September 2018

Accepted 2nd November 2018

DOI: $10.1039 / \mathrm{c} 8 \mathrm{ra07300g}$

rsc.li/rsc-advances

\section{Deep eutectic solvents as a new kind of dispersive solvent for dispersive liquid-liquid microextraction $\dagger$}

\author{
Andrey Shishov, (D)* Natalia Volodina, Daria Nechaeva, (D) Svetlana Gagarinova \\ and Andrey Bulatov
}

In this paper, deep eutectic solvents (DESs) were investigated as a new kind of dispersive solvent for effective dispersive liquid-liquid microextraction (DLLME) for the first time. It was established that the DESs can increase the extraction efficiency compared to conventional polar, water-miscible organic dispersive solvents.
One of the most common sample pretreatment methods used in chemical analysis is liquid-liquid extraction (LLE), which is characterized by simplicity, reliability and compatibility with modern analytical instrumentation. However, conventional LLE is time consuming and requires large amounts of organic solvents. Currently miniaturization, which means the significant reduction of the volume of hazardous organic solvents, is the main demand, which corresponds to the concept of green analytical chemistry (GAC). ${ }^{1}$ Thus, development of miniaturized, eco-friendly, less expensive, faster and simpler sample pretreatment methods is a very important task in GAC.

Dispersive liquid-liquid microextraction (DLLME) is an attractive tool for the miniaturized sample pretreatment of a wide range of sample matrices (water samples, ${ }^{2}$ biological fluids, ${ }^{3}$ foods, ${ }^{4}$ etc) introduced in 2006 by Rezaee et al. ${ }^{5}$ The DLLME has grown increasingly popular (ESM Fig. 1) due to its simplicity, low cost and high availability. In the DLLME, a cloudy solution of fine organic droplets is formed when a mixture of extraction solvent (immiscible with water) and dispersive solvent (miscible with both water and the extractant solvent) is rapidly injected into an aqueous phase. In the DLLME, large surface contact between fine organic droplets and sample speeds up the processes regarding mass-transfer of analytes from aqueous phase to organic phase which greatly enhances extraction efficiency. The DLLME can be coupled with various analytical methods (HPLC-UV, ${ }^{6}$ HPLC-MS, ${ }^{7}$ GC-MS, ${ }^{8}$ spectrophotometry, ${ }^{9}$ electrochemical methods, ${ }^{10}$ AAS, ${ }^{11}$ $e t c)$ and can by automated based on flow systems. ${ }^{12-14}$ However, one of the main disadvantages of the conventional DLLME is in need of using an additional dispersive organic solvent that can

Department of Analytical Chemistry, Institute of Chemistry, Saint-Petersburg University, Saint Petersburg State University, SPbSU, SPbU, 7/9 Universitetskaya nab., St. Petersburg 199034, Russia. E-mail: a.y.shishov@spbu.ru

$\dagger$ Electronic supplementary information (ESI) available: Number of publications on dispersive liquid-liquid microextraction (DLLME) for the past 11 years. See DOI: $10.1039 / \mathrm{c} 8 \mathrm{ra} 07300 \mathrm{~g}$ increase solubility of the hydrophobic analytes in an aqueous phase reducing the extraction efficiency. ${ }^{15}$ Moreover, relatively large volumes of hazardous dispersive organic solvents such as methanol, acetonitrile, acetone, ${ }^{16}$ etc are required to disperse extraction solvents in aqueous phase.

In this work, a new phenomenon - fast dispersion of extraction solvent in aqueous phase in the presence of deep eutectic solvent (DES) resulting excellent analyte extraction was established for the first time and used for the DLLME of bisphenol-A (BPA) as a proof-of-concept analyte in beverages followed by its determination by high-performance liquid chromatography with fluorescence detection (HPLC-FLD).

BPA is a polyphenol compound used in the manufacturing of polycarbonates-based plastic packaging. It is known, that BPA as a carcinogen can be found in food products stored in the plastic packaging. ${ }^{17}$ Therefore, monitoring of BPA's content in foods and drinks is an important task for analytical chemistry.

Recently, DESs are presently getting increased attention from researchers in analytical chemistry as high-efficiency extraction solvents. ${ }^{18}$ DESs are formed by mixing two or three

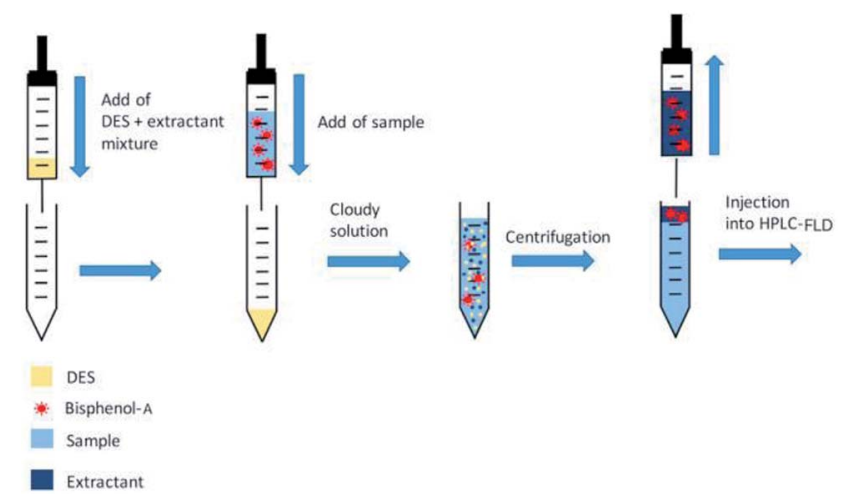

Fig. 1 Scheme of dispersive liquid-liquid microextraction using DES as dispersive solvent. 
cheap components that are capable of interacting through the formation of hydrogen bonds, to form a eutectic mixture with a melting point lower than that of each individual component. ${ }^{19}$ DESs are regarded as a cheap alternative to ionic liquids. DES are easy to be produced and cheaper due to lower cost of the raw materials. ${ }^{20}$

In this research, long-chain alcohols (heptanol, octanol, decanol) as extraction solvents mixed with various DESs as dispersive solvents were studied. DESs were prepared by mixing tetrabutylammonium bromide $(\mathrm{TBABr})$ as a hydrogen bond acceptor and ethylene glycol, glycerol, acetic and formic acids as donors of hydrogen bond in the molar ratio of $1: 2$ at $80^{\circ} \mathrm{C}^{21}$ The obtained DESs were viscous liquids at ambient condition. The kinematic viscosity values of the DESs were estimated using micro capillary viscometers $\left(17.1,15.7,154.5\right.$ and $471 \mathrm{~mm}^{2} \mathrm{~s}^{-1}$ at $30{ }^{\circ} \mathrm{C}$ for the DES based on TBABr and formic acid, acetic acid, ethylene glycol and glycerol, respectively). Unlike conventional DLLME, the aqueous sample phase was injected into an extraction mixture of dispersive solvent (DES) and extraction solvent by a syringe because the extraction mixtures were viscous and their aspiration into the syringe was difficult. As result fast dissolution and decomposition of the DES and dispersion of the extraction solvent in aqueous sample phase was observed resulting fast extraction of BPA into extraction solvent droplets Fig. 1. After centrifugation (2 min, $4000 \mathrm{rpm}$ ) the upper phase was collected and injected into a HPLC-FLD system (LC-20 Prominence liquid chromatograph system, Shimadzu, Japan). Chromatographic separation was performed using a Zorbax Bonus-RP column (Agilent, $2500 \times 2.1 \mathrm{~mm}, 3.5$ $\mu \mathrm{m})$. Mobile phase was deionized water-methanol $(1: 3, \mathrm{v} / \mathrm{v})$.

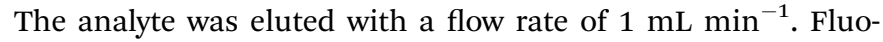
rescence detection was carried out with excitation and emission at 230 and $305 \mathrm{~nm}$, respectively.

Criterion for the choice of DES as a dispersive solvent was the ability to be mixed well with the aqueous phase and extraction solvent as well to provide the maximum extraction efficiency and reproducibility. All DESs studied were mixed with longchain alcohols used as extractants as well as with aqueous phase forming homogeneous solutions. The maximum peak areas were found for the DESs based on acetic and formic acids (Fig. 2A). This fact can be explained by suppression of analyte dissociation in acidic media resulting more effective analyte extraction. During aqueous sample injection into extraction mixture dissolution and decomposition of the DES is observed resulting acidification of the aqueous phase. Moreover, TBABr acts as salting-out agent and promotes BPA extraction. It should be pointed out that the DES based on the formic acid was characterized by less RSD value due to its less viscosity and it was chosen for future studies.

The selection of the extraction solvent is important in the DLLME to achieve good extraction efficiency and efficient analyte enrichment. Long-chain alcohols as green extraction solvents were studied for extraction of BPA. In the experiments, $5 \mathrm{~mL}$ of aqueous BPA solution $\left(1 \mu \mathrm{g} \mathrm{L}^{-1}\right)$ was injected into extraction mixture containing $250 \mu \mathrm{L}$ of long-chain alcohol and $1 \mathrm{~g}$ of DES prepared (in all cases viscous liquid phase). It was found, that the use of octanol provided maximum extraction efficiency (Fig. 2B).

The volume of extraction solvent may result in preconcentration or dilution of extracted analyte. The volume of extraction
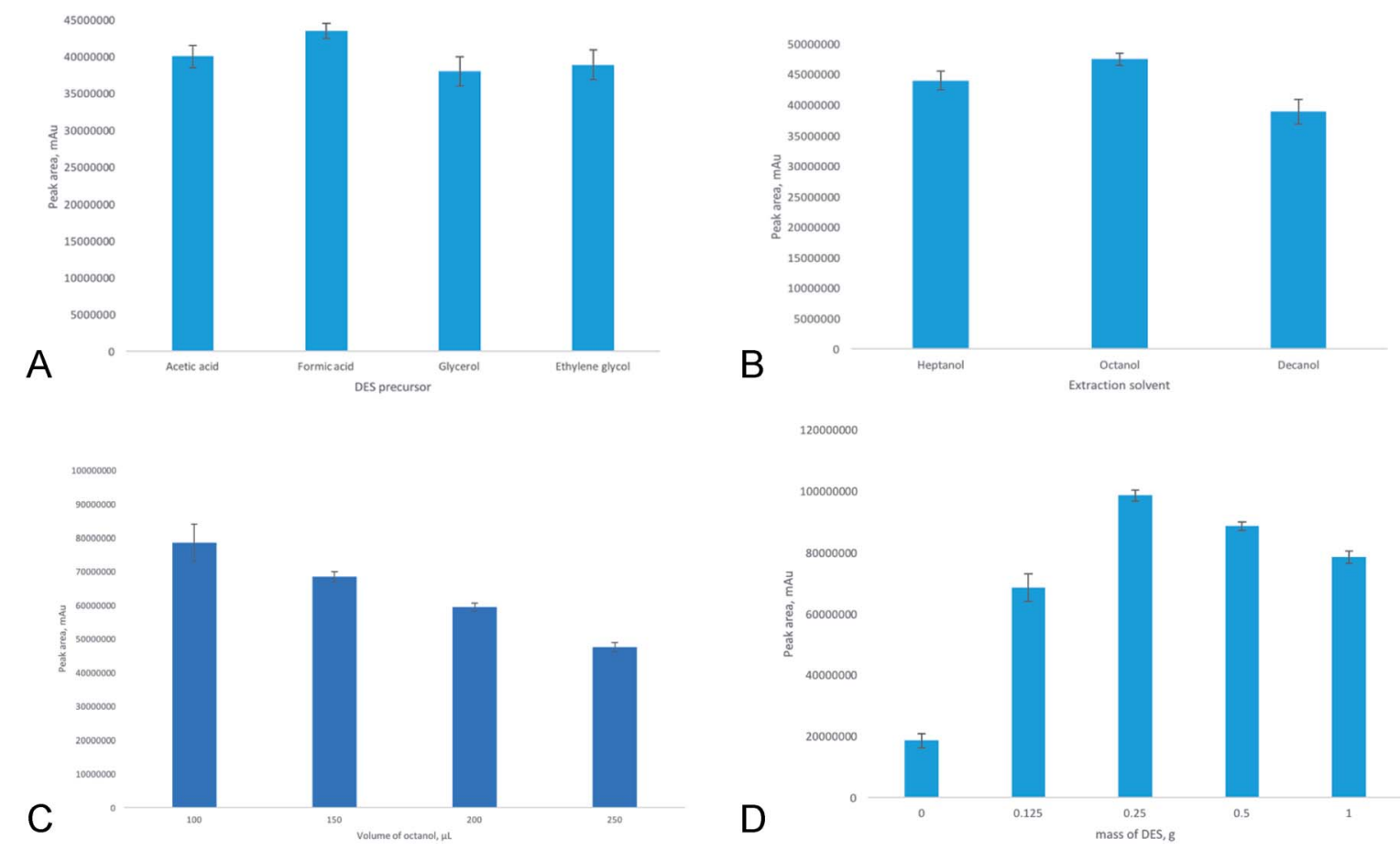

Fig. 2 (A.) Effect of the eutectic solvent precursor on the extraction efficiency of bisphenol-A, (B.) effect of the type of extraction solvent on the extraction efficiency of bisphenol-A, (C.) the effect of the volume of extraction solvent on the extraction efficiency of bisphenol-A, (D.) effect of DES mass on the extraction efficiency of bisphenol-A. 
solvent should be as small as possible to achieve high enrichment factor. $5 \mathrm{~mL}$ of aqueous BPA solution $\left(1 \mathrm{mg} \mathrm{L^{-1 }}\right)$ was injected into extraction mixture containing $1 \mathrm{~g}$ of DES (TBABrformic acid) and extraction solvent (octanol). The octanol volume was varied from 100 to $250 \mu \mathrm{L}$. It was shown (Fig. 2C), that maximum peak area was observed for $100 \mu \mathrm{L}$ of octanol. However, in this case it was difficult to withdraw the upper organic phase resulting poor reproducibly $(\mathrm{RSD}=15 \%)$. The use of $150 \mu \mathrm{L}$ of octanol was characterized by satisfactory extraction efficiency and better reproducibly $(\mathrm{RSD}=4 \%)$. Thus, $150 \mu \mathrm{L}$ of octanol was chosen as the optimum volume.

To reduce the consumption of DES, the effect of its amount on the extraction efficiency was also studied. As can be seen from the data (Fig. 2D), amount of DES in the extraction mixture significantly influenced on the extraction process. The analytical signal was increased with DES mass decreasing up to $250 \mathrm{mg}$. BPA extraction into octanol phase without DES and in the presence of TBABr $(190 \mathrm{mg})$ in aqueous phase was characterized by poor extraction efficiency (extraction recovery (ER) values were $32 \%$ and $41 \%$, respectively). ER was calculated as ER $=\left(C_{\text {sop }} V_{\text {sop }} / C_{0} V_{0}\right) \times 100 \%$, were $C_{\text {sop }}$ and $C_{0}$ - analyte concentration in the separated organic phase and in the initial sample phase, respectively; $V_{\text {sop }}$ and $V_{0}$ - separated organic phase and sample volumes, respectively. If the DES content was higher than $250 \mathrm{mg}$ dilution effect of extractant phase was predominant and solubility of analyte in aqueous phase was increased and as result analytical signal was reduced. This fact was confirmed by the HPLC-FLD determination of BPA in aqueous phase after the DLMME. Thus, DES mass of $250 \mathrm{mg}$ was chosen as the optimum value.

After optimization of extraction conditions, the proposed approach was compared with the DLMME procedure using conventional polar, water-miscible organic dispersive solvents. For this purpose, the extraction mixture containing $150 \mu \mathrm{L}$ of octanol and $250 \mu \mathrm{L}$ of dispersive solvent (acetone, acetonitrile, methanol, ethanol or isopropyl alcohol) was injected into $5 \mathrm{~mL}$ of aqueous BPA solution $\left(1 \mathrm{mg} \mathrm{L}^{-1}\right)$ by the syringe. Moreover, formic acid was also studied as dispersive solvent. After centrifugation organic phase was analyzed. As can be seen from the obtained results (Fig. 3), the proposed approach provided significantly higher extraction efficiency. It was established, that ER values obtained for DES, acetone, acetonitrile, methanol, ethanol, isopropyl alcohol and formic acid were 95\%, $49 \%, 61 \%, 58 \%, 55 \%, 59 \%$ and $47 \%$, respectively. The obtained results shown that polar, water-miscible organic dispersive solvents increase solubility of BPA in an aqueous phase.

To evaluate the proposed procedure, some relevant parameters such as enrichment factor (EF), linearity correlation coefficient $\left(R^{2}\right)$, precision, limit of detection (LOD) and limit of quantification (LOQ) were determined in the optimized condition. EF value was 33. EF was estimated as the ratio between the analyte concentration in the separated organic phase $\left(C_{\text {sop }}\right)$ and the initial concentration of analyte $\left(C_{0}\right)$ in the sample phase: EF $=C_{\mathrm{sop}} / C_{0}$. The calibration graph was linear over the concentration ranges from 0.001 to $1 \mathrm{mg} \mathrm{L}^{-1}$ with linearity correlation coefficient of 0.996 . The LOD and LOQ were calculated as three and ten times the standard deviation of the blank test $(n=10)$,

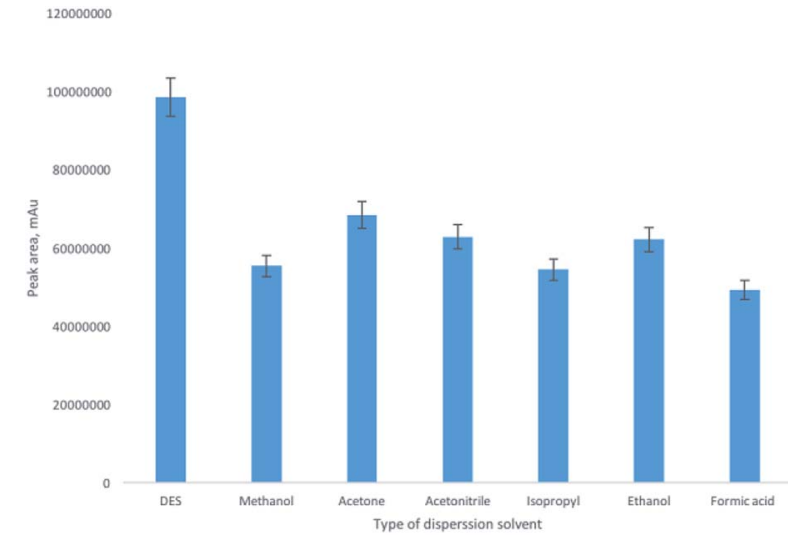

Fig. 3 Comparison of the extraction efficiency using DES and polar organic solvents as dispersive solvents.

respectively. The LOD was $0.0003 \mathrm{mg} \mathrm{L}^{-1}$ and the LOQ was $0.0009 \mathrm{mg} \mathrm{L}^{-1}$. The method's repeatability expressed as RSD ( $n$ $=5$ ) was $4.0 \%$ and $2 \%$ at $0.001 \mathrm{mg} \mathrm{L}^{-1}$ and $1 \mathrm{mg} \mathrm{L}^{-1}$, respectively.

The developed procedure was used for the determination of BPA in three beverages samples (still water, sparkling water and Orange juice) purchased from local supermarkets (Saint Petersburg, Russia). As can be seen from the obtained data, BPA was found in sparkling water sample stored in a plastic bottle (Table 1). Accuracy and reliability of the resulting information were further studied by add-found method. The recoveries obtained for each of the samples were within of $93-110 \%$ range which is considered acceptable for this type of samples.

In conclusion, really effective DLMME approach using DES as a dispersive solvent was developed and successfully applied for the HPLC-FLD determination of BPA in beverages. Above all, DES allowed to significantly improve the extraction efficiency in comparison with conventional polar, water-miscible organic dispersive solvents. The benefits observed during the dispersion process are related to the action of the DES and individual components of the DES system. During aqueous sample injection into homogeneous octanol - DES mixture decomposition of the DES is observed resulting dispersion of octanol phase and extraction of analyte. In this case, TBABr as quaternary ammonium salt containing bromide ion can act as salting-out agent and promote mass transfer between the two phases. Comparative studies have indicated that the developed approach is a reliable analytical tool with wide potential applications in

Table 1 The results of bisphenol-A determination in beverages $(n=4$; $P=0.95)$

\begin{tabular}{llll}
\hline & \multicolumn{2}{l}{ Concentration, $\mathrm{mg} \mathrm{L}^{-1}$} & \\
\cline { 2 - 3 } Sample & Added & Found & Recovery, \% \\
\hline Still water & 0 & $<$ LOD & - \\
Sparkling water & 0.01 & $0.0094 \pm 0.0001$ & 94 \\
& 0 & $0.020 \pm 0.002$ & - \\
Orange juice & 0.01 & $0.028 \pm 0.002$ & 93 \\
& 0 & $<$ LOD & - \\
& 0.01 & $0.011 \pm 0.002$ & 110
\end{tabular}


analysis of various sample matrixes and it has the opportunity to be coupled with other instrumental methods.

\section{Conflicts of interest}

There are no conflicts to declare.

\section{Acknowledgements}

Andrey Shishov gratefully acknowledges financial support for research from Russian Scientific Foundation (project no. 18-7300111). HPLC-FLD assay was performed at the Chemistry Educational Centre of Research Park of St. Petersburg State University.

\section{Notes and references}

1 S. Armenta, S. Garrigues and M. de la Guardia, TrAC, Trends Anal. Chem., 2008, 27, 497-511.

2 M. Rezaee, Y. Assadi, M.-R. Milani Hosseini, E. Aghaee, F. Ahmadi and S. Berijani, J. Chromatogr. A, 2007, 66, 8-86.

3 H. A. Mashayekhi, P. Abroomand-Azar, M. Saber-Tehrani and S. W. Husain, Chromatographia, 2010, 71, 517-521.

4 H. Abdolmohammad-Zadeh and G. H. Sadeghi, Talanta, 2010, 81, 778-785.

5 M. Rezaee, Y. Assadi, M.-R. Milani Hosseini, E. Aghaee, F. Ahmadi and S. Berijani, J. Chromatogr. A, 2006, 1116, 1-9.

6 X. W. Lai, D. L. Sun, C. Q. Ruan, H. Zhang and C. L. Liu, J. Sep. Sci., 2014, 37, 92-98.

7 R. S. Zhao, X. Wang, J. Sun, C. Hu and X. K. Wang, Microchim. Acta, 2011, 174, 145-151.

8 A. Melo, S. C. Cunha, C. Mansilha, A. Aguiar, O. Pinho and I. M. P. L. V. O. Ferreira, Food Chem., 2015, 38, 3552-3559.
9 N. Pourreza, S. Rastegarzadeh and A. Larki, Talanta, 2015, 134, 24-29.

10 E. Fernández, L. Vidal, D. Martín-Yerga, M. D. C. Blanco, A. Canals and A. Costa-García, Talanta, 2015, 135, 34-40.

11 N. N. Meeravali, K. Madhavi and S. J. Kumar, J. Anal. At. Spectrom., 2016, 31, 1582-1589.

12 A. N. Anthemidis and K.-I. G. Ioannou, Talanta, 2009, 79, 86-91.

13 V. Andruch, C. Cecilia Acebal, J. Skrlikova, H. Sklenarova, P. Solich, I. S. Balogh, F. Billes and L. Kocurova, Microchem. J., 2012, 100, 77-82.

14 A. Bulatov, K. Medinskaia, D. Aseeva, S. Garmonov and L. Moskvin, Talanta, 2015, 133, 66-70.

15 J. Regueiro, M. Llompart, C. Garcia-Jares, J. C. GarciaMonteagudo and R. Cela, J. Chromatogr. A, 2008, 1190, 2738.

16 N. Campillo, P. Viñas, J. Šandrejová and V. Andruch, Appl. Spectrosc. Rev., 2017, 52, 267-415.

17 N. Salgueiro-González, S. Castiglioni, E. Zuccato, I. TurnesCarou, P. López-Mahía and S. Muniategui-Lorenzo, Anal. Chim. Acta, 2018, 1024, 39-51.

18 A. Shishov, A. Bulatov, M. Locatelli, S. Carradori and V. Andruch, Microchem. J., 2017, 135, 33-38.

19 A. P Abbott, D. Boothby, G. Capper, D. L. Davies and R. K. Rasheed, J. Am. Chem. Soc., 2004, 126, 9142-9147.

20 Y. Cui, C. Li, J. Yin, S. Li, Y. Jia and M. Bao, Design, synthesis and properties of acidic deep eutectic solvents based on choline chloride, J. Mol. Liq., 2017, 236, 338-343.

21 R. Yusof, E. Abdulmalek, K. Sirat and M. B. A. Rahman, Molecules, 2014, 19, 8011-8026. 